\title{
COHERENT VACUUM-UV SOURCES USING \\ NONLINEAR OPTICS
}

\begin{abstract}
By
H. B. Puell, H. Scheingraber and C.R. Vidal Max Planck Institut fur Extraterrestische Physik, 8046 Garching, Germany
\end{abstract}

For investigating diatomic and triatomic molecules of astrophysical interest in the vacuum UV using the methods of laser spectroscopy we have set up several systems for generating coherent vacuum UV sources. Since we are interested in tunable and narrow banded $\left(0.1-0.01 \mathrm{~cm}^{-1}\right)$ sources it is advantageous to employ the methods of nonlinear optics.

Recently, experiments of the third harmonic generatuon in the $\mathrm{Rb}-\mathrm{Xe}$ system have been performed and a quantitative interpretation up to the highest conversion efficiencies of a few percent has been given (1). On the basis of these experiments we have investigated the optimum conditions for third harmonic generation in isotropic media. It has been possible to solve the third order problem analytically and it can be shown that the intensity conversion efficiency depends in the limit of large input intensities only on the wavelength dependent nonlinear susceptibilities of the nonlinear medium. For a Gaussian beam it can be shown that a Na-Xe system, for example, is capable of providing an energy conversion efficiency of up to $40 \%$ for the Nd:glass laser.

Resonant and nonresonant third harmonic generation and frequency mixing in two component systems has been performed using a frequency doubled high power ruby laser and flash-lamp and nitrogen laser pumped dye lasers. So far, these systems have covered the wavelength of $1157 \AA$ (sixth harmonic of ruby) and a range of about 1400 to $2000 \AA$. Photon densities have been achieved which greatly exceed the corresponding values of existing synchrotrons. Detailed studies of the phase matching for parallel and focussed beams have been carried out and the intensity dependence and the influence of the relevant absorption cross sections have been investigated.

1. H. B. Puell, K. Spanner, W. Falkenstein, W. Kaiser and C. R. Vidal. Phys. Rev. A14, 2240 (1976). 